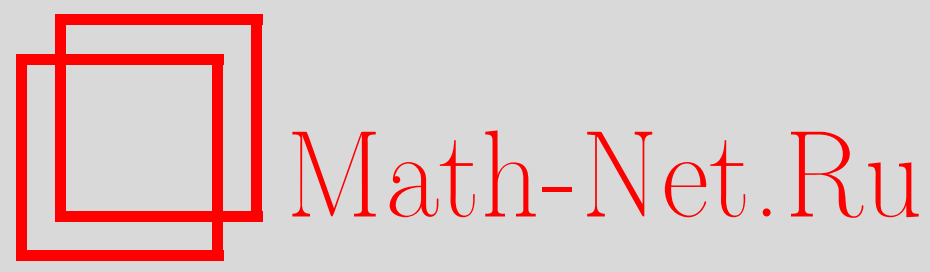

А. А. Бободжанов, В. Ф. Сафонов, Сингулярно возмущенные нелинейные интегро-дифференциальные системы с быстро изменяющимися ядрами, Матем. заметки, 2002, том 72, выпуск 5, 654-664

DOI: https://doi.org/10.4213/mzm453

Использование Общероссийского математического портала Math-Net.Ru подразумевает, что вы прочитали и согласны с пользовательским соглашением http://www . mathnet.ru/rus/agreement

Параметры загрузки:

IP : 54.224 .135 .184

26 апреля 2023 г., 18:21:40

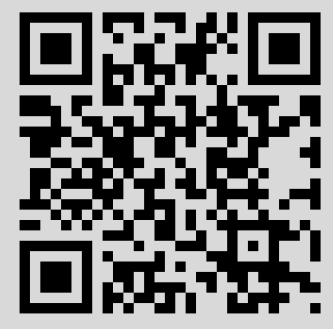




\title{
СИНГУЛЯРНО ВОЗМУЩЕННЫЕ НЕЛИНЕЙНЫЕ ИНТЕГРО-ДИФФЕРЕНЦИАЛЬНЫЕ СИСТЕМЫ С БЫСТРО ИЗМЕНЯЮШИМИСЯ ЯДРАМИ
}

\author{
А. А. Бободжанов, В. Ф. Сафонов
}

\begin{abstract}
Рассматриваются нелинейные сингулярно возмущенные интегро-дифференциальные уравнения с быстро изменяющимися ядрами. Предполагается, что спектр предельного оператора лежит в замкнутой левой полуплоскости $\operatorname{Re} \lambda \leqslant 0$. Разрабатывается алгоритм, позволяюший получать регуляризованные (по С. А. Ломову) асимптотические решения как в нерезонансном, так и в резонансном случае. При развитии алгоритма существенно используется аппарат регуляризации интегральных операторов с быстро изменяющимися ядрами, разработанный ранее авторами для линейных интегральных и интегро-дифференциальных систем. Обоснование алгоритма и доказательство существования решения исходной нелинейной задачи проводится с помощью метода Ньютона для операторных уравнений.
\end{abstract}

Библиограффия: 6 названий.

1. Введение. Интегро-дифференциальным сингулярно возмущенньм системам посвящено много работ (см., например, [1], [2]). В этих работах подробно обсуждаются различные случаи асимптотического поведения решений этих систем (при стремлении малого параметра к нулю) и предлагаются соответствующие алгоритмы построения асимптотических решений. В зависимости от поведения спектра предельного оператора применим тот или иной алгоритм. Если точки спектра лежат в открытой левой полуплоскости, то используется, как правило, метод пограничных функций Васильевой-Бутузова [3], модифицированньй применительно к интегро-дифференциальным уравнениям М.И. Иманалиевым [2]. Если точки спектра при некоторых значениях независимой переменной попадают на мнимую ось, то асимптотический анализ интегро-дифференциальных уравнений методом погранфункций становится невозможным. В этом случае прибегают к другим методам, например, к методу регуляризации Ломова [1]. Однако этот метод применялся в основном к линейньм задачам с медленно изменяюшимися коэффициентами. В настоящей работе рассматриваются нелинейные системы вида

$$
\begin{gathered}
\varepsilon \frac{d y}{d t}=A(t) y+\int_{0}^{t} \exp \left(\frac{1}{\varepsilon} \int_{s}^{t} \mu(\theta) d \theta\right) K(t, s) y(s, \varepsilon) d s+\varepsilon f(y, t)+h(t), \\
y(0, \varepsilon)=y^{0}, \quad t \in[0, T],
\end{gathered}
$$

с быстро изменяюшимися ядрами. Здесь скалярная функция $\mu(t)$, называемая спектральным значением ядра интегрального оператора, может не обращаться в нуль 
на отрезке $[0, T]$. Она индуцирует в решении задачи (1) дополнительные быстро изменяющиеся компоненты. Даже в случае медленно изменяющихся ядер $(\mu(t) \equiv 0)$ задача (1) при наличии чисто мнимых точек спектра $\left\{\lambda_{j}(t)\right\}$ оператора $A(t)$ представляет определенный интерес, так как в цитированных выше работах она не рассматривалась. Здесь впервые предпринимается такая попытка. При этом предполагается, что $\mu(t) \neq 0$ $\forall t \in[0, T]$. Однако развиваемьй ниже алгоритм очевидным образом модифицируется на случай $\mu(t) \equiv 0$.

2. Регуляризация задачи (1). Уточним условия, при которых будет рассматриваться система (1). Будем предполагать, что

1) $\lambda_{n+1} \equiv \mu(t) \in C^{\infty}[0, T], K(t, s) \in C^{\infty}\left(0 \leqslant s \leqslant t \leqslant T, \mathbb{C}^{n^{2}}\right), h(t) \in C^{\infty}\left([0, T], \mathbb{C}^{n}\right)$, $A(t) \in C^{\infty}\left([0, T], \mathbb{C}^{n^{2}}\right), f(y, t)-$ многочлен по $y$,

$$
f(y, t)=\sum_{0 \leqslant|m| \leqslant l} f^{(m)}(t) y^{(m)}
$$

с коэффициентами $f^{(m)}(t) \in C^{\infty}\left([0, T], \mathbb{C}^{n}\right), 0 \leqslant|m| \leqslant l<\infty$;

2) $\lambda_{i}(t) \neq \lambda_{j}(t), i \neq j, \lambda_{i}(t) \neq 0(\forall t \in[0, T], i, j=1, \ldots, n+1)$;

3) $\operatorname{Re} \lambda_{i}(t) \leqslant 0, \operatorname{Re} \lambda_{n+1}(t)<0(\forall t \in[0, T], i=1, \ldots, n)$;

4) равенство $(m, \lambda(t)) \equiv m_{1} \lambda_{1}(t)+\cdots+m_{n+1} \lambda_{n+1}(t)=\lambda_{j}(t)$ (при $|m| \geqslant 2$ и $j \in\{1,2, \ldots, n+1\})$ либо не имеет места ни при каком $t \in[0, T]$, либо выполняется при всех $t \in[0, T]$.

Следуя методу Ломова [1], вводим регуляризирующие функции

$$
\tau_{j}=\frac{1}{\varepsilon} \int_{0}^{t} \lambda_{j}(s) d s \equiv \frac{\psi(t)}{\varepsilon}, \quad j=1, \ldots, n+1 .
$$

Для функции $\widetilde{y}(t, \tau, \varepsilon)$, удовлетворяющей условию

$$
\left.\widetilde{y}(t, \tau, \varepsilon)\right|_{\tau=\psi / \varepsilon} \equiv y(t, \tau, \varepsilon), \quad \tau=\left(\tau_{1}, \ldots, \tau_{n+1}\right), \quad \psi=\left(\psi_{1}, \ldots, \psi_{n+1}\right),
$$

где $y(t, \varepsilon)$ - решение системы (1), получим следующую задачу:

$$
\begin{aligned}
\varepsilon \frac{\partial \widetilde{y}}{\partial t}+\sum_{j=1}^{n+1} \lambda_{j}(t) \frac{\partial \widetilde{y}}{\partial \tau_{j}}= & A(t) \widetilde{y}+\int_{0}^{t} \exp \left(\frac{1}{\varepsilon} \int_{s}^{t} \mu(\theta) d \theta\right) K(t, s) \widetilde{y}\left(s, \frac{\psi(s)}{\varepsilon}, \varepsilon\right) d s \\
& +\varepsilon f(\widetilde{y}, t)+h(t), \quad \widetilde{y}(0,0, \varepsilon)=y^{0} .
\end{aligned}
$$

Однако в (3) не произведена регуляризация интегрального члена

$$
I \widetilde{y}(t, \varepsilon)=\int_{0}^{t} \exp \left(\frac{1}{\varepsilon} \int_{s}^{t} \mu(\theta) d \theta\right) K(t, s) \widetilde{y}\left(s, \frac{\psi(s)}{\varepsilon}, \varepsilon\right) d s .
$$

Для этой цели необходимо ввести класс $M_{\varepsilon}$, асимптотически инвариантньй (при $\varepsilon \rightarrow$ $+0)$ относительно оператора $I$ (см. $[1$, с. 62$])$. В линейном случае $(f(y, t) \equiv 0)$ в качестве такого класса выступает пространство вектор-функций, "натянутых" на единицу и экспоненты $\exp \left(\psi_{j}(t) / \varepsilon\right)$, порождаемые спектром $\left\{\lambda_{j}(t)\right\}$ предельного оператора $A(t)$. В нелинейном случае это пространство должно быть существенно расширено, так как нелинейность $f(y, t)$ индуцирует в решениях сингулярно возмушенных задач экспоненты $\exp \left(\frac{1}{\varepsilon}(m, \lambda(t))\right.$ измерения $|m| \equiv m_{1}+\cdots+m_{n+1}$ вьше первого (см., например, [4]).

Следуя [4], введем сначала пространство $U$, описьваемое следующим образом. 
ОПРЕДЕЛЕНИЕ 1. Будем говорить, что вектор-функиия $y(t, \tau)=\left\{y_{1}, \ldots, y_{n}\right\} n p u-$ надлежит пространству $U$, если она представляется в виде суммы

$$
y(t, \tau)=y^{(0)}(t)+\sum_{1 \leqslant|m| \leqslant N}^{\prime} y^{(m)}(t) e^{(m, \tau)}, \quad N=N_{y}<\infty
$$

с коэффициентами $y^{(0)}(t), y^{(m)}(t) \in C^{\infty}\left([0, T], \mathbb{C}^{n}\right)$, не содержащей резонансных экспонент, т.е. таких экспонент $\exp (m, \tau)$ измерения $|m| \geqslant 2$, для которых при некоторых $j \in\{1,2, \ldots, n+1\}$ и $t \in[0, T]$ выполняется равенство

$$
(m, \lambda(t)) \equiv m_{1} \lambda_{1}(t)+\cdots+m_{n+1} \lambda_{n+1}(t)=\lambda_{j}(t)
$$

(факт отсутствия в (5) резонансных экспонент отмечен штрихом над суммой).

В качестве класса $M_{\varepsilon}$ возьмем пространство $\left.U\right|_{\tau=\psi(t) / \varepsilon}$. Ниже будет показано, что образ $I y(t, \tau)$ произвольного элемента класса $M_{\varepsilon}$ разлагается в ряд по степеням $\varepsilon$, сходящийся асимптотически при $\varepsilon \rightarrow+0$. Это и будет означать, что класс $M_{\varepsilon}$ асимптотически инвариантен относительно интегрального оператора (4).

Подставляя (5) в интегральньй оператор (4), будем иметь

$$
\begin{aligned}
\operatorname{Iy}(t, \tau) & =\sum_{1 \leqslant|m| \leqslant N}^{\prime} \exp \left(\frac{1}{\varepsilon} \int_{0}^{t} \lambda_{n+1}(\theta) d \theta\right) \int_{0}^{t} \exp \left(\frac{1}{\varepsilon} \int_{0}^{s}\left(m-e_{n+1}, \lambda(\theta)\right) d \theta\right) k^{(m)}(t, s) d s \\
& +\exp \left(\frac{1}{\varepsilon} \int_{0}^{t} \lambda_{n+1}(\theta) d \theta\right) \int_{0}^{t} \exp \left(-\frac{1}{\varepsilon} \int_{0}^{s} \lambda_{n+1}(\theta) d \theta\right) k^{(0)}(t, s) d s
\end{aligned}
$$

где обозначено $k^{(m)}(t, s) \equiv K(t, s) y^{(m)}(s), m \geqslant 0$. Надо показать, что стоящие здесь интегралы

$$
I^{(m)}(t, \varepsilon)=\exp \left(\frac{1}{\varepsilon} \int_{0}^{t} \lambda_{n+1}(\theta) d \theta\right) \int_{0}^{t} \exp \left(\frac{1}{\varepsilon} \int_{0}^{s}\left(m-e_{n+1}, \lambda(\theta)\right) d \theta\right) k^{m}(t, s) d s
$$

где $|m| \geqslant 0, m \neq e_{n+1}$, разлагаются в асимптотические ряды по степеням $\varepsilon$. Сначала заметим, что при указанных в (6) мультииндексах $m$ выражение $\left(m-e_{n+1}, \lambda(t)\right)$ не обращается в нуль на отрезке $[0, T]$. При $m=0$ и $m=e_{i}(i \neq n+1)$ это вытекает из вьписанных вьше условий 2). При $|m| \geqslant 2$ в сумме (5) отсутствуют резонансные экспоненты, а значит,

$$
\left(m-e_{n+1}, \lambda(t)\right) \equiv(m, \lambda(t))-\lambda_{n+1}(t) \neq 0 \quad \forall t \in[0, T]
$$


Указанное неравенство позволяет применить в (7) операцию интегрирования по частям:

$$
\begin{aligned}
I^{(m)}(t, \varepsilon) & \varepsilon \exp \left(\frac{1}{\varepsilon} \int_{0}^{t} \lambda_{n+1}(\theta) d \theta\right) \int_{0}^{t} \frac{k^{(m)}(t, s)}{\left(m-e_{n+1}, \lambda(s)\right)} d \exp \left(\frac{1}{\varepsilon} \int_{0}^{s}\left(m-e_{n+1}, \lambda(\theta)\right) d \theta\right) \\
= & \varepsilon \exp \left(\frac{1}{\varepsilon} \int_{0}^{t} \lambda(\theta) d \theta\right) \\
\times & {\left[\left(\frac{k^{(m)}(t, t)}{\left(m-e_{n+1}, \lambda(t)\right)} \exp \left(\frac{1}{\varepsilon} \int_{0}^{t}\left(m-e_{n+1}, \lambda(\theta)\right) d \theta\right)-\frac{k^{(m)}(t, 0)}{\left(m-e_{n+1}, \lambda(0)\right)}\right)\right.} \\
& \left.-\int_{0}^{t} \exp \left(\frac{1}{\varepsilon} \int_{0}^{s}\left(m-e_{n+1}, \lambda(\theta)\right) d \theta\right) \frac{\partial}{\partial s}\left(\frac{k^{(m)}(t, s)}{\left(m-e_{n+1}, \lambda(s)\right)}\right) d s\right] \\
= & \left(\frac{k^{(m)}(t, t)}{\left(m-e_{n+1}, \lambda(t)\right)} \exp \left(\frac{1}{\varepsilon} \int_{0}^{t}(m, \lambda(\theta)) d \theta\right)\right. \\
& \left.\left.-\frac{k^{(m)}(t, 0)}{\left(m-e_{n+1}, \lambda(0)\right)} \exp \left(\frac{1}{\varepsilon} \int_{0}^{t} \lambda_{n+1}(\theta)\right) d \theta\right)\right) \\
& \left.-\varepsilon \exp \left(\frac{1}{\varepsilon} \int_{0}^{t} \lambda_{n+1}(\theta)\right) d \theta\right) \\
\times & \int_{0}^{t} \exp \left(\frac{1}{\varepsilon} \int_{0}^{s}\left(m-e_{n+1}, \lambda(\theta)\right) d \theta\right) \frac{\partial}{\partial s}\left(\frac{k^{(m)}(t, s)}{\left(m-e_{n+1}, \lambda(s)\right)}\right) d s .
\end{aligned}
$$

Продолжая этот процесс далее, получим ряд

$$
\begin{aligned}
I^{(m)}(t, \varepsilon)= & \sum_{\nu=0}^{\infty}(-1)^{(\nu)} \varepsilon^{\nu+1}\left[\left(I_{m}^{\nu} k^{(m)}(t, s)\right)_{s=t} \exp \left(\frac{1}{\varepsilon} \int_{0}^{t}(m, \lambda(\theta)) d \theta\right)\right. \\
& \left.-\left(I_{m}^{\nu} k^{(m)}(t, s)\right)_{s=0} \exp \left(\frac{1}{\varepsilon} \int_{0}^{t} \lambda_{n+1}(\theta) d \theta\right)\right], \quad|m| \geqslant 0, \quad m \neq e_{n+1},
\end{aligned}
$$

где введены операторы

$$
I_{m}^{0}=\frac{1}{\left(m-e_{n+1}, \lambda(s)\right)}, \quad I_{m}^{\nu}=\frac{1}{\left(m-e_{n+1}, \lambda(s)\right)} \frac{\partial}{\partial s} I_{m}^{\nu-1}, \quad \nu \geqslant 1 .
$$

В силу вьполнения условий 1)-3) ряды, стоящие в правой части (8), будут асимптотически сходиться (при $\varepsilon \rightarrow+0)$ к интегралам $I^{(m)}(t, \varepsilon)$, что доказывается точно так же, как и аналогичное утверждение в линейном случае (см. [5]). Но тогда из (7) следует, что образ $\left.I y(t, \tau)\right|_{\tau=\psi(t) / \varepsilon}$ интегрального оператора (4) системы (1) разлагается в асимптотический ряд

$$
\begin{aligned}
I y(t, \tau)= & \exp \left(\frac{1}{\varepsilon} \int_{0}^{t} \lambda_{n+1}(\theta) d \theta\right) \int_{0}^{t} k^{e_{n+1}}(t, s) d s \\
& +\sum_{\substack{0 \leqslant|m| \leqslant N \\
m \neq e_{n+1}}}^{\prime} \sum_{\nu=0}^{\infty}(-1)^{\nu} \varepsilon^{\nu+1}\left[\left(I_{m}^{\nu} k^{(m)}(t, s)\right)_{s=t} \exp \left(\frac{1}{\varepsilon} \int_{0}^{t}(m, \lambda(\theta)) d \theta\right)\right. \\
& \left.-\left(I_{m}^{\nu} k^{(m)}(t, s)\right)_{s=0} \exp \left(\frac{1}{\varepsilon} \int_{0}^{t} \lambda_{n+1}(\theta) d \theta\right)\right] .
\end{aligned}
$$


Тем самьм показано, что класс $M_{\varepsilon}$ асимптотически инвариантен относительно интегрального оператора $I$.

Если теперь ряд

$$
\widetilde{y}(t, \tau, \varepsilon)=\sum_{k=0}^{\infty} \varepsilon^{k} y_{k}(t, \tau) \equiv \sum_{k=0}^{\infty} \varepsilon^{k}\left(\sum_{0 \leqslant|m| \leqslant N_{k}}^{\prime} y^{(m)}(t) e^{(m, \tau)}\right),
$$

сходящийся асимптотически (при $\varepsilon \rightarrow+0)$ равномерно по $(t, \tau) \in[0, T] \times \Pi$, то его образ $I \widetilde{y}(t, \tau, \varepsilon)$ будет, очевидно, также представляться асимптотическим рядом (при $\varepsilon \rightarrow+0)$. Это позволяет получить окончательное расширение интегрального оператора $I$ следуюшим образом.

Для произвольного элемента (5) пространства $U$ можно записать, что

$$
I y(t, \tau)=R_{0} y(t, \tau)+\sum_{\nu=0}^{\infty}\left(R_{\nu+1} y(t, \tau)\right) \varepsilon^{\nu+1}
$$

где через $R_{\nu}$ обозначены операторы порядка (действующие в $U$ ), определяемые формулами (см. [4])

$$
\begin{aligned}
& R_{0} y(t, \tau)=e^{\tau}+1 \int_{0}^{t} k^{e_{n+1}}(t, s) d s \\
& R_{\nu+1} y(t, \tau)=(-1)^{\nu} \sum_{\substack{0 \leqslant|m| \leqslant N \\
m \neq e_{n+1}}}^{\prime}\left[\left(I_{m}^{\nu} k^{(m)}(t, s)\right)_{s=t} e^{(m, \tau)}-\left(I_{m}^{\nu} k^{(m)}(t, s)\right)_{s=0} e^{\tau_{n+1}}\right], \\
& \nu \geqslant 1, \quad \tau=\frac{\psi(t)}{\varepsilon} .
\end{aligned}
$$

С учетом этих формул результат подстановки (10) в интеграл $I \widetilde{y}$ можно записать в виде

$$
I \widetilde{y}=\left.\sum_{r=0}^{\infty} \varepsilon^{r} \sum_{\substack{s=0 \\ r-s \geqslant 0}}^{r} R_{r-s} y_{s}(t, \tau)\right|_{\tau=\psi(t) / \varepsilon} .
$$

Формальным расширением оператора $I$ назовем оператор

$$
\widetilde{I} \widetilde{y}(t, \tau, \varepsilon) \equiv \widetilde{I}\left(\sum_{k=0}^{\infty} \varepsilon^{k} y_{k}(t, \tau)\right)=\sum_{r=0}^{\infty} \varepsilon^{r} \sum_{\substack{s=0 \\ r-s \geqslant 0}}^{r} R_{r-s} y_{s}(t, \tau) .
$$

Хотя этот оператор определен формально, его сужение $\left.\widetilde{I} \widetilde{y}(t, \tau, \varepsilon)\right|_{\tau=\psi(t) / \varepsilon}$ на функциях $y(t, \tau, \varepsilon) \in C([0, T], \Pi)$, представимых рядами (10) (сходящимися при $\varepsilon \rightarrow+0$ равномерно по $(t, \tau))$, имеет вполне конкретный смысл:

$$
\left.\widetilde{I} \widetilde{y}(t, \tau, \varepsilon)\right|_{\tau=\psi(t) / \varepsilon}=I \widetilde{y}(t, \tau, \varepsilon) .
$$

Это равенство означает, что сужение (при $\tau=\psi(t) / \varepsilon)$ образа функции $\widetilde{y}(t, \tau, \varepsilon)$ расширения оператора $\widetilde{I}$ является асимптотическим рядом, сходяшимся при $\varepsilon \rightarrow+0$ к вполне конкретной функции $I \widetilde{y}(t, \tau, \varepsilon)$. 
Теперь можно записать в окончательной форме систему, расширенную по отношению к исходной (1):

$$
\varepsilon \frac{\partial \widetilde{y}}{\partial t}+L_{0} \widetilde{y}-\widetilde{I} \widetilde{y}(t, \tau, \varepsilon)-\varepsilon f(\widetilde{y}, t)=h(t), \quad \widetilde{y}(0,0, \varepsilon)=y^{0}
$$

где

$$
L_{0} \widetilde{y} \equiv \sum_{j=1}^{n+1} \lambda_{j}(t) \frac{\partial \widetilde{y}}{\partial \tau_{j}}-A(t) \widetilde{y}
$$

Это расширение определено по крайней мере в классе рядов (10), сходящихся асимптотически при $\varepsilon \rightarrow+0$ (равномерно по $(t, \tau) \in[0, T] \times \Pi$, где $\Pi=\left\{\tau: \operatorname{Re} \tau_{j} \leqslant 0, j=1, \ldots\right.$, $n+1\})$, что вполне достаточно для развития алгоритма построения асимптотического решения исходной задачи (1).

3. Разрешимость итерационных систем. Будем определять решение задачи (11) в виде ряда (10). Для коэффициентов этого ряда получим следующие задачи:

$$
\begin{array}{ll}
L y_{0}(t, \tau) \equiv L_{0} y_{0}-R_{0} y_{0}=h(t), & y_{0}(0,0)=y^{0} \\
L y_{1}(t, \tau)=-\frac{\partial y_{0}}{\partial t}+\widehat{f}\left(y_{0}(t, \tau), t\right)+R_{1} y_{0}, & y_{1}(0,0)=0 \\
L y_{2}(t, \tau)=-\frac{\partial y_{1}}{\partial t}-\frac{\partial f\left(y_{0}, t\right)}{\partial y} y_{1}+R_{1} y_{1}+R_{2} y_{0}, & y_{2}(0,0)=0
\end{array}
$$

Здесь значком “-” обозначена операция вложения, ставящая в соответствие произвольной сумме

$$
z(t, \tau)=\sum_{|m| \geqslant 0}^{N} z^{(m)}(t) e^{(m, t)}=\sum_{|m| \geqslant 0}^{N} z^{(m)}(t) e^{(m, \tau)}+\sum_{j=1}^{n+1} \sum_{\substack{\left|m^{j}\right| \geqslant 2 \\\left(m^{j}, \lambda(t)\right) \equiv \lambda_{j}(t)}} z^{\left(m^{j}\right)}(t) e^{\left(m^{j}, \tau\right)}
$$

(в которой могут присутствовать резонансные экспоненты) элемент $\widehat{z}(t, \tau)$ пространства $U$, вычисляемьй следующим образом:

$$
\widehat{z}(t, \tau)=\sum_{|m| \geqslant 0}^{\prime} z^{(m)}(t) e^{(m, t)}+\sum_{j=1}^{n+1} \sum_{\substack{\left|m^{j}\right| \geqslant 2 \\\left(m^{j}, \lambda(t)\right) \equiv \lambda_{j}(t)}} z^{\left(m^{j}\right)} e^{\tau_{j}}
$$

Решение задачи (12) вычисляем в виде элемента пространства $U$

$$
y_{0}(t, \tau)=\sum_{0 \leqslant|m| \leqslant N_{0}}^{\prime} y^{(m)}(t) e^{(m, \tau)} .
$$


Подставляя (15) в (12) и приравнивая отдельно свободные члены и коэффициенты при одинаковых экспонентах, получим системы

$$
\begin{gathered}
-A(t) y_{0}^{(0)}(t)=h(t), \\
{\left[\lambda_{j}(t) I-A(t)\right] y_{0}^{e_{j}}(t)=0, \quad j=1, \ldots, n,} \\
{\left[\lambda_{n+1}(t) I-A(t)\right] y_{0}^{e_{n+1}}(t)-\int_{0}^{t} K(t, s) y_{0}^{e_{n+1}}(s) d s=0,} \\
{[(m, \lambda(t)) I-A(t)] y^{(m)}(t)=0, \quad|m| \geqslant 2, \quad(m, \lambda(t)) \neq \lambda_{j}(t), \quad j=1, \ldots, n .}
\end{gathered}
$$

Поскольку $\lambda_{n+1}(t) \neq \lambda_{j}(t), j=1, \ldots, n, \operatorname{det} A(t) \neq 0, \operatorname{det}([(m, \lambda(t)) I-A(t)]) \neq 0$ $\forall t \in[0, T]$, то системы $(16),(18),(19)$ имеют следуюшие решения:

$$
y_{0}^{(0)}(t)=-A^{-1}(t) h(t), \quad y_{0}^{e_{n+1}}(t) \equiv 0, \quad y^{(m)}(t) \equiv 0
$$

Система (17) имеет решения вида

$$
y_{0}^{e_{j}}(t)=\alpha_{0}^{e_{j}}(t) \varphi_{j}(t), \quad j=1, \ldots, n,
$$

где $\alpha_{0}^{e_{j}}(t) \in C^{\infty}\left([0, T], \mathbb{C}^{1}\right)$ - пока произвольные функции, $j=1, \ldots, n$. При этом решение (15) системы (12) записывается в форме

$$
y_{0}(t, \tau)=-A^{-1}(t) h(t)+\sum_{j=1}^{n} \alpha_{0}^{e_{j}}(t) \varphi_{j}(t) e^{\tau_{j}}
$$

Подчиняя его начальному условию, найдем, что

$$
\sum_{j=1}^{n} \alpha_{0}^{e_{j}}(0) \varphi_{j}(0)=A^{-1}(0) h(0)+y^{0},
$$

откуда (умножая скалярно обе части на $\chi(0))$ вычисляем

$$
\alpha_{0}^{e_{j}}(0)=\left(A^{-1}(0) h(0)+y^{0}, \chi(0)\right) .
$$

Перейдем теперь к задаче (13). Подставляя в (13) решение (22) системы (12), будем иметь

$$
\begin{aligned}
& L y_{1}(t, \tau) \\
& =-\dot{y}_{0}^{(0)}(t)-\sum_{j=1}^{n}\left(\dot{\alpha}_{0}^{e_{j}}(0) \varphi_{j}(t)+\alpha_{0}^{e_{j}}(t) \dot{\varphi}_{j}(t)\right) e^{\tau_{j}}+f_{0}(t) \\
& +\sum_{\substack{|m| \geqslant 2 \\
(m, \lambda(t)) \neq \lambda_{i}(t) \\
j=1, \ldots, n+1}} f^{(m)}\left(\alpha_{0}^{e_{1}}, \ldots, \alpha_{0}^{e_{n}}, t\right) e^{(m, \tau)} \\
& +\sum_{i=1}^{n+1} f^{e_{i}}\left(\alpha_{0}^{e_{1}}, \ldots, \alpha_{0}^{e_{n}}, t\right) e^{\tau_{i}}+\left(I_{0}^{0} K(t, s) y_{0}^{(0)}(s)\right)_{s=t}-\left(I_{0}^{0} K(t, s) y_{0}^{(0)}(s)\right)_{s=0} e^{\tau_{n+1}} \\
& +\sum_{j=1}^{n}\left[\left(I_{e_{j}}^{0} K(t, s) \alpha_{0}^{e_{j}}(s) \varphi(s)\right)_{s=t} e^{\tau_{j}}-\left(I_{e_{j}}^{0} K(t, s) \alpha_{0}^{e_{j}}(s) \varphi(s)\right)_{s=0} e^{\tau_{n+1}}\right],
\end{aligned}
$$


где учтено, что

$$
\begin{gathered}
\widehat{f}\left(y_{0}(t, \tau), t\right)=f_{0}(t)+\sum_{\substack{i=1 \\
+}}^{n+1} f^{e_{i}}\left(\alpha_{0}^{e_{1}}, \ldots, \alpha_{0}^{e_{n}}, t\right) e^{\tau_{i}} \\
+\sum_{\substack{|m| \geqslant 2 \\
(m, \lambda(t)) \neq \lambda_{i}(t) \\
i=1, \ldots, n+1}} f^{(m)}\left(\alpha_{0}^{e_{1}}, \ldots, \alpha_{0}^{e_{n}}, t\right) e^{(m, \tau)}, \\
R_{1} y(t, \tau) \equiv \sum_{\substack{0 \leqslant|m| \leqslant N \\
m \neq e_{n+1}}}^{\prime}\left[\left(I_{m}^{0} K(t, s) y^{(m)}(s)\right)_{s=t} e^{(m, \tau)}-\left(I_{m}^{0} K(t, s) y^{(m)}(s)\right)_{s=0} e^{\tau_{n+1}}\right]
\end{gathered}
$$

и обозначено $y_{0}^{(0)}(t) \equiv-A^{-1}(t) h(t)$.

Определяя решение системы $(24)$ в виде суммы

$$
y_{1}(t, \tau)=\sum_{|m| \geqslant 0}^{\prime} y_{1}^{(m)}(t) e^{(m, \tau)}
$$

получим следуюшие системы для коэффищиентов $y_{1}^{(m)}(t)$ :

$$
\begin{aligned}
& -A(t) y_{1}^{(0)}(t)=-\dot{y}_{0}^{(0)}(t)+f_{0}(t)+\left(I_{0}^{0} K(t, s) y_{0}^{(0)}(0)\right)_{s=t} \\
& {\left[\lambda_{j}(t) I-A(t)\right] y_{1}^{e_{j}}(t)=-\dot{\alpha}_{0}^{e_{j}}(t) \varphi_{j}(t)-\alpha_{0}^{e_{j}}(t) \dot{\varphi}_{j}(t)} \\
& +\left(I_{e_{j}}^{0} K(t, s) \alpha_{0}^{e_{j}}(s) \varphi_{j}(s)\right)_{s=t}+f^{e_{j}}\left(\alpha_{0}^{e_{1}}, \ldots, \alpha_{0}^{e_{n}}, t\right), \quad j=1, \ldots, n, \\
& {\left[\lambda_{n+1}(t) I-A(t)\right] y_{1}^{e_{n}+1}(t)-\int_{0}^{t} K(t, s) y_{1}^{e_{n+1}}(s) d s=-\left(I_{0}^{0} K(t, s) y_{0}^{(0)}(s)\right)_{s=0}} \\
& -\sum_{j=1}^{n}\left(I_{e_{j}}^{0} K(t, s) \alpha_{0}^{e_{j}}(s) \varphi(s)\right)_{s=0}+f^{e_{n+1}}\left(\alpha_{0}^{e_{1}}, \ldots, \alpha_{0}^{e_{n}}, t\right), \\
& {[(m, \lambda(t)) I-A(t)] y_{1}^{m}(t)=f^{m}\left(\alpha_{0}^{e_{1}}, \ldots, \alpha_{0}^{e_{n}}, t\right),} \\
& |m| \geqslant 2, \quad(m, \lambda(t)) \neq \lambda_{j}(t), \quad j=1, \ldots, n+1 .
\end{aligned}
$$

Как и в предыдущем случае, устанавливаем, что системы (26), (28) и (29) имеют единственные решения в классе $C^{\infty}\left([0, T], \mathbb{C}^{n}\right)$. Для разрешимости системы $(27)$ в указанном классе необходимо и достаточно, чтобы

$$
\begin{gathered}
\left(-\dot{\alpha}_{0}^{e_{j}}(t) \varphi_{j}(t)-\alpha_{0}^{e_{j}}(t) \dot{\varphi}_{j}(t)+\frac{K(t, t) \alpha_{0}^{e_{j}}(t) \varphi_{j}(t)}{\lambda_{j}(t)-\lambda_{n+1}(t)}+f e_{j}\left(\alpha_{0}^{e_{1}}, \ldots, \alpha_{0}^{e_{n}}, t\right), \chi_{j}(t)\right) \equiv 0 \\
j=1, \ldots, n
\end{gathered}
$$

или

$$
\begin{gathered}
\dot{\alpha}_{0}^{e_{j}}=-\left[\left(\dot{\varphi}_{j}(t), \chi(t)\right)-\frac{\left(K(t, t) \varphi_{j}(t), \chi(t)\right)}{\lambda_{j}(t)-\lambda_{n+1}(t)}\right] \alpha_{0}^{e_{j}}+\left(f^{e_{j}}\left(\alpha_{0}^{e_{1}}, \ldots, \alpha_{0}^{e_{n}}, t\right), \chi_{j}(t)\right)=0 \\
j=1, \ldots, n
\end{gathered}
$$


При этом функции $\alpha_{0}^{e_{j}}(t)$ должны удовлетворять начальным условиям (23). Система $(30),(23)$ в резонансном случае является нелинейной системой дифференциальных уравнений, а значит, ее разрешимость на отрезке $[0, T]$ не гарантирована. Если, например, спектр $\left\{\lambda_{j}(t)\right\}$ матрицы $A(t)$ в фиксированной точке $t \in[0, T]$ расположен по одну сторону от некоторой прямой $\pi$, проходящий через нуль комплексной плоскости $\lambda$, и на ней нет точек $\lambda_{j}(t)$, то система является треугольной. В этом случае уравнения (30) последовательно интегрируются и, значит, их разрешимость на отрезке $[0, T]$ становися очевидной. В других случаях расположения спектра $\lambda_{j}(t)$ относительно мнимой оси треугольность системы (30) нарушается. Однако в любом случае существует некоторая бирациональная замена переменных, позволяющая понизить порядок системы и свести исследование проблемы разрешимости в целом для более простой системы дифференциальных уравнений. Мы не будем обсуждать здесь эту проблему. Потребуем, чтобы система $(30),(23)$ имела решение в классе $C^{\infty}\left([0, T], \mathbb{C}^{n}\right)$. В этом случае функции $\alpha_{0}^{e_{j}}(t)$, входящие в решение (10) системы (12), будут полностью вычислены, а сама система (12) будет иметь единственное решение в пространстве $U$. При этом будет найдено решение (25) системы (13) с точностью до элементов ядра $\alpha_{1}^{e_{1}}(t) e^{\tau_{1}}+\cdots+\alpha_{1}^{e_{n}} e^{\tau_{n}}$. Поиск функций $\alpha_{1}^{e_{1}}(t), \ldots \alpha_{1}^{e_{n}}(t)$, входящих в указанное ядро, осуществляется по той же схеме, что и поиск функций $\alpha_{0}^{e_{j}}(t)$. При этом для $\alpha_{i}^{e_{j}}(t)$ уже получается линейная система дифференциальных уравнений, разрешимость которой на отрезке $[0, T]$ гарантирована гладкостью входящих в нее коэффициентов. Сформулируем соответствующий результат в виде теоремы.

ТЕОРемА 1. Пусть выполнены условия 1)-4) и задача (30), (23) разрешима на отрезке $[0, T]$. Тогда все итерационные задачи (12), (13), (14), .. однозначно разрешимы в классе $U$ (при их последовательном решении).

4. Асимптотическая сходимость формальных решений. Построив решения $y_{0}(t, \tau), \ldots, y_{N}(t, \tau)$ задач $(12),(13),(14), \ldots$ в пространстве $U$, составим частичную сумму

$$
S_{N}(t, \tau, \varepsilon)=\sum_{k=0}^{N} \varepsilon^{k} y_{k}(t, \tau)
$$

Обозначим сужение этой суммы при $\tau=\psi(t) / \varepsilon$ через $y_{\varepsilon N}(t)$. Имеет место следующее утверждение.

Лемма 1. Пусть выполнены условия теоремы 1. Тогда функиия $y_{\varepsilon N}(t)$ удовлетворяет задаче (1) с точностью до членов, содержащих $\varepsilon^{N+1}$, т.е.

$$
\begin{array}{r}
\varepsilon \frac{d y_{\varepsilon N}}{d t}=A(t) y_{\varepsilon N}(t)+\int_{0}^{t} \exp \left(\frac{1}{\varepsilon} \int_{s}^{t} \mu(\theta) d \theta\right) K(t, s) y_{\varepsilon N}(s, \varepsilon) d s \\
+\varepsilon f\left(y_{\varepsilon N}, t\right)+h(t)+\varepsilon^{N+1} R(t, \varepsilon), \quad y_{\varepsilon N}(0)=y^{0}
\end{array}
$$

әде $\|R(t, \varepsilon)\|_{C[0, T]} \leqslant \bar{R}, \bar{R}>0$ - постоянная, не зависящая от $\varepsilon \in\left(0, \varepsilon_{0}\right], \varepsilon_{0}>0$ достаточно мало. 
ДокАЗАТЕЛЬСтво. Подставляя $y_{0}(t, \tau), \ldots, y_{N}(t, \tau)$ в первые $N$ систем $(12),(13)$, $(14), \ldots$ соответственно, получим тождества. Умножим их на $1, \varepsilon, \ldots, \varepsilon^{N}$ соответственно и сложим. Будем иметь

$$
\begin{aligned}
& L_{0}\left(y_{0}+\varepsilon y_{1}+\cdots+\varepsilon^{N} y_{N}\right)-R_{0}\left(y_{0}+\varepsilon y_{1}+\cdots+\varepsilon^{N} y_{N}\right) \\
& \equiv h(t)-\varepsilon\left(\frac{\partial y_{0}}{\partial t}+\varepsilon \frac{\partial y_{1}}{\partial t}+\cdots+\varepsilon^{N-1} \frac{\partial y_{N-1}}{\partial t}\right) \\
&+\varepsilon R_{1} y_{0}+\varepsilon^{2}\left(R_{1} y_{1}+R_{2} y_{0}\right)+\varepsilon^{N}\left(R_{1} y_{N-1}+\cdots+R_{N} y_{0}\right) \\
&+\varepsilon \widehat{f}\left(y_{0}, t\right)+\varepsilon^{2} \frac{\partial f\left(y_{0}, t\right)}{\partial y} y_{1}+\cdots+\varepsilon^{N} \widehat{P}_{N}\left(y_{0}, \ldots, y_{N-1}, t\right) .
\end{aligned}
$$

Произведем здесь сужение при $\tau=\psi(t) / \varepsilon$; при этом учтем, что выполняются тождества

$$
\begin{gathered}
\left.\left.\sum_{0 \leqslant|m| \leqslant k} z^{(m)}(t) e^{(m, \tau)}\right|_{\tau=\psi(t) / \varepsilon} \equiv \sum_{0 \leqslant|m| \leqslant k} z^{(m)}(t) e^{(m, \tau)}\right|_{\tau=\psi(t, \varepsilon)}, \\
\left.L_{0} v(t, \tau, \varepsilon)\right|_{\tau=\psi(t) / \varepsilon} \equiv \varepsilon \frac{d v(t, \psi(t) / \varepsilon, \varepsilon)}{d t}-A(t) v\left(t, \frac{\psi(t)}{\varepsilon}, \varepsilon\right)-\varepsilon \frac{\partial v(t, \psi(t) / \varepsilon, \varepsilon)}{\partial t} .
\end{gathered}
$$

Будем иметь

$$
\begin{aligned}
\varepsilon \frac{d y_{\varepsilon N}}{d t}-A(t) y_{\varepsilon N}(t)= & \varepsilon^{N+1} \frac{\partial y_{N}(t, \psi)}{\partial t}+h(t)+\sum_{r=0}^{N} \varepsilon^{r} \sum_{\substack{s=0 \\
r-s \geqslant 0}}^{r} R_{r-s} y_{s}(t, \psi) \\
& +\varepsilon\left[f\left(y_{0}, t\right)+\varepsilon \frac{\partial f\left(y_{0}, t\right)}{\partial y} y_{1}+\cdots+\varepsilon^{N-1} P_{N}\left(y_{0}, \ldots, y_{N-1}, t\right)\right],
\end{aligned}
$$

или

$$
\begin{aligned}
\varepsilon \frac{d y_{\varepsilon N}}{d t}= & A(t) y_{\varepsilon N}(t)+\int_{0}^{t} \exp \left(\frac{1}{\varepsilon} \int_{s}^{t} \mu(\theta) d \theta\right) K(t, s) y_{\varepsilon N}(s) d s \\
& +\varepsilon f\left(y_{\varepsilon N}(t), t\right)+h(t)+\varepsilon^{N+1} \frac{\partial y_{N}(t, \psi)}{\partial t} \\
& -\left[\int_{0}^{t} \exp \left(\frac{1}{\varepsilon} \int_{s}^{t} \mu(\theta) d \theta\right) K(t, s) y_{\varepsilon N}(s) d s-\sum_{r=0}^{N} \varepsilon^{r} \sum_{\substack{s=0 \\
r-s \geqslant 0}}^{r} R_{r-s} y_{s}(t, \psi)\right] \\
& -\varepsilon\left[f\left(y_{\varepsilon N}(t), t\right)-f\left(y_{0}, t\right)-\frac{\partial f\left(y_{0}, t\right)}{\partial y} y_{1}-\cdots-\varepsilon^{N-1} P_{N}\left(y_{0}, \ldots, y_{N-1}, t\right)\right] .
\end{aligned}
$$

По определению операторов $R_{k}$ выражение в первой квадратной скобке последнего тождества представляется в виде $\varepsilon^{N+1} M_{1}(t, \varepsilon)$, а по построению задач $(12),(13), \ldots$ выражение во второй квадратной скобке представляется в виде $\varepsilon^{N+1} M_{2}(t, \varepsilon)$, где

$$
\left\|M_{i}\right\|_{C[0, T]} \leqslant \text { const } \quad \forall \varepsilon \in\left(0, \varepsilon_{0}\right], \quad i=1,2 .
$$

Обозначая

$$
R(t, \varepsilon) \equiv M_{1}(t, \varepsilon)+M_{2}(t, \varepsilon)+\frac{\partial y_{N}(t, \psi)}{\partial t}
$$


получим утверждение леммы.

При обосновании асимптотической сходимости формального решения $y_{\varepsilon}(t) \mathrm{k}$ точному $y(t, \varepsilon)$ используется следующее утверждение (см. [6]), касающееся разрешимости операторного уравнения

$$
P_{\varepsilon}(u)=0 .
$$

Tеорема 2. Пусть оператор $P_{\varepsilon}$ действует из банахова пространства $B_{1} \boldsymbol{\theta}$ банахово пространство $B_{2}$ и имеет в некотором шаре $\left\{\left\|u-u_{0}\right\| \leqslant r\right\} \subset B_{1} \partial в е$ непрерывные производные. Пусть также существует оператор $\Gamma_{\varepsilon} \equiv\left[P_{\varepsilon}^{\prime}\left(u_{0}\right)\right]^{-1}$ и выполнены условия:

1) $\left\|\Gamma_{\varepsilon}\right\| \leqslant c_{1} \varepsilon^{-k}$;

2) $\left\|P_{\varepsilon}\left(u_{0}\right)\right\| \leqslant c_{2} \varepsilon^{m}(m>2 k)$;

3) $\left\|P_{\varepsilon}^{\prime \prime}(u)\right\| \leqslant c_{3}$.

Тогда уравнение (33) имеет при достаточно малых $\varepsilon>0\left(\varepsilon \in\left(0, \varepsilon_{0}\right]\right)$ решение $u_{*} \in B_{1}$, удовлетворяющее неравенству

$$
\left\|u_{*}-u_{0}\right\|_{B_{1}} \leqslant c \varepsilon^{m-k}
$$

Здесь $c, c_{1}, c_{2}, c_{3}$ - некоторые положительные постоянные, не зависящие от $\varepsilon \in$ $\left(0, \varepsilon_{0}\right]$.

Получен следующий основной результат.

ТЕОРЕМА 3. Пусть выполнены условия 1)-4) и задача (30), (23) разрешима в целом на отрезке $[0, T]$. Тогда при достаточно мальх $\varepsilon \in\left(0, \varepsilon_{0}\right]$ исходная задача (1) имеет в классе $C^{1}\left([0, T], \mathbb{C}^{n}\right)$ единственное решение $y(t, \varepsilon)$ и справедлива оценка

$$
\left\|y(t, \varepsilon)-y_{\varepsilon N}(t)\right\|_{C[0, T]} \leqslant C_{N} \varepsilon^{N+1}, \quad N=0,1, \ldots,
$$

где постоянная $C_{N}>0$ не зависит от $\varepsilon$ при $\left(0, \varepsilon_{0}\right]$.

\section{СПИСОК ЦИТИРОВАННОЙ ЛИТЕРАТУРЫ}

[1] Ломов С. А. Введение в общую теорию сингулярных возмущений. М.: Наука, 1981.

[2] Иманалиев М. И. Методы решения нелинейных обратных задач и их приложения. Фрунзе, 1977.

[3] Васильева А. Б., Бутузов В. Ф. Асимптотические разложения решений сингулярно возмущенных уравнений. М.: Наука, 1973.

[4] Сафонов В.Ф. Метод регуляризации для сингулярно возмущенных систем нелинейных дифференциальных уравнений // Изв. АН СССР. Сер. матем. 1979. Т. 43. № 3. С. 628-653.

[5] Сафонов В.Ф., Туйчиев О. Д. Регуляризация сингулярно возмущенных интегральньх уравнений с быстро изменяющимся ядром и их асимптотика // Дифференц. уравнения. 1997. Т. 33. № 9. С. 1199-1211.

[6] Срубщик Л. С., Юдович В. И.О применении метода Ньютона в задачах асимптотического интегрирования нелинейных уравнений // Тезисы докладов Всесоюзной межвузовской конференции по применению методов функционального анализа к решению нелинейных задач. Баку, 1975. С. 103. 